Table 1

\begin{tabular}{lcccc}
\hline & $\begin{array}{c}\text { Stable Angina } \\
\mathrm{n}=335\end{array}$ & $\begin{array}{c}\text { Unstable angina nont S-T } \\
\mathrm{n}=804\end{array}$ & $\begin{array}{c}\text { Myocardial Infarction } \\
\mathrm{n}=536\end{array}$ & $\begin{array}{c}\text { Healthy } \\
\mathrm{N}=680\end{array}$ \\
\hline L2- L4 & $-2.6 \pm 11^{*}$ & $-2.9 \pm 1.4^{\star *}$ & $-3.1 \pm 1.1^{\star \star}$ & $-0.6 \pm 0.2$ \\
Prox. Femur Neck & $-1.8 \pm 0.5^{\star}$ & $-2.6 \pm 1.4^{\star *}$ & $-2.5 \pm 1.6^{* *}$ & $-1.14 \pm 0.26$ \\
Prox. Femur Total & $-2.7 \pm 1.48^{* \star}$ & $-2.7 \pm 0.2^{* \star}$ & $-2.7 \pm 1.25^{* \star}$ & $-1.05 \pm 0.5$ \\
\hline
\end{tabular}

${ }^{*} p<0.01{ }^{* *} p<0.001$.

deteriorated than cortical 3.Correlation between $\mathrm{T}$ Score values and clinical forms of Atherosclerosis were not observed 4 . The best understanding of interrelations of mechanisms could point out the right direction for the simultaneous therapy against both targets - Osteoporosis and Atherosclerosis 5. Therefore the expectation of establishing novelty direction among other subtypes of the Medical Specialties as a Preventive Gerontology can be realistic

Disclosure of Interest: None declared

DOI: 10.1136/annrheumdis-2017-eular.1110

\section{AB0847 ACCURATE ASSESSMENT OF FEMORAL ECHOSOUND APPROACH PERFORMANCE THROUGH DXA ERROR ANALYSIS}

M. Muratore ${ }^{1}$, F. Conversano ${ }^{2}$, P. Pisani ${ }^{2}$, M. Peccarisi ${ }^{3}$, A. Greco ${ }^{3}$, T. De Marco $^{3}$, E. Quarta ${ }^{1}$, L. Quarta ${ }^{1}$, A. Grimaldi ${ }^{1}$, S. Casciaro ${ }^{2} .{ }^{1} \mathrm{OU}$ of Rheumatology Galateo Hospital, San Cesario di Lecce, ASL-LE; ${ }^{2}$ National Research Council, Institute of Clinical Physiology; ${ }^{3}$ Echolight Spa, LECCE, Italy

Background: Currently, the accepted "gold standard" method for bone mineral density (BMD) measurement and osteoporosis diagnosis related to the reference axial anatomical sites is dual-energy X-ray absorptiometry (DXA). However, actual DXA effectiveness is limited by several factors, including intrinsic accuracy uncertainties and specific errors in patient positioning, image segmentation, and post-acquisition data analysis, as documented by very recent literature [1]. This may affect the comparative evaluation of the effectiveness of novel diagnostic methods whose validation studies adopt DXA outcomes as standard reference to assess diagnostic performance.

Objectives: To assess the impact of DXA errors on the performance of an innovative ultrasound parameter for osteoporosis diagnosis on the femoral site, known as Osteoporosis Score (OS).

Methods: 202 patients aged in 46-75 years underwent two diagnostic investigations on the femoral neck: a conventional femoral DXA and an echographic scan performed by employing the innovative EchoSound technology [2]. Initially, the performance of the OS parameter was evaluated considering all the available DXA reports as reference in the data analysis, and calculating the corresponding accuracy in patient classification (osteoporotic, osteopenic, or healthy) and the correlation coefficient between the DXA-measured BMDs and the OS-derived $B M D$ values. At a later stage, the DXA errors were taken into account by performing a strict quality control on DXA reports: all those cases affected by a typical inaccuracy [1] were excluded from the analysis and the actual diagnostic accuracy of the EchoSound technology was re-assessed by analysing only the reliable DXA reports. Intra- and inter-operator repeatability of OS-derived BMD values were also measured in a group of patients.

Results: A diagnostic accuracy of $84.4 \%(r=0.78, p<0.001)$ was obtained for the EchoSound approach when all the DXA reports were included in the analysis. In the second part of the study, 61 out of the initial $202(30.4 \%)$ patients were excluded from the analysis because their DXA reports were affected by specific errors. An actual diagnostic accuracy of $94.2 \%$ was then obtained on the remaining 141 patients, together with a high correlation between DXA-measured BMDs and OS-derived BMD values $(r=0.88, p<0.001)$. Intra- and inter-operator repeatability of OS-derived BMD values, expressed in terms of the root mean square coefficient of variation (RMS-CV), resulted equal to $0.29 \%$ and $0.34 \%$ for intra- and inter-operator variability, respectively, therefore documenting a very good measurement repeatability.

Conclusions: Undetected DXA errors had an impact on the accuracy evaluation of EchoSound femoral neck densitometry, causing an underestimation of OS performance in osteoporosis diagnosis. The quality control analysis on DXA reports can be useful to study the actual performance of different ultrasonic methods that considered routine DXA reports as the gold standard reference.

References:

[1] Eur Radiol 2015;25:1504

[2] UMB 2015;41:281.

Acknowledgements: Work partially funded by FESR PO Apulia Region 2007-13 - Action 1.2.4 (grant n. 3Q5AX31: ECHOLIGHT Project).

Disclosure of Interest: M. Muratore: None declared, F. Conversano Shareholder of: Echolight spa, a National Research Council spin-off that may or may not benefit from results of this study, P. Pisani: None declared, M. Peccarisi Employee of: Echolight Spa, A. Greco Employee of: Echolight Spa, T. De Marco Employee of: Echolight Spa, E. Quarta: None declared, L. Quarta: None declared, A. Grimaldi: None declared, S. Casciaro Shareholder of: Echolight spa, a National Research Council spin-off that may or may not benefit from results of this study DOI: 10.1136/annrheumdis-2017-eular.4843

\section{AB0848 DENTAL PANORAMIC RADIOGRAPHY AS A TOOL FOR IDENTIFICATION OF OSTEOPOROSIS: AMONG TUNISIAN WOMEN}

M. Brahem ${ }^{1}$, M. Jguirim ${ }^{1}$, M. Khemiss ${ }^{2}$, I. Chaabani ${ }^{2}$, E. Chebil $^{3}$, M. Younes ${ }^{4}$, T. Ben Alaya ${ }^{2}$, M. Ben Khelifa ${ }^{2}$, I. Bejia ${ }^{1}$, M. Touzi ${ }^{1}$, S. Zrour ${ }^{1}$, N. Bergaoui ${ }^{1}$. ${ }^{1}$ Rheumatology; ${ }^{2}$ Stomatology, Fattouma Bourguiba Hospital Monastir, Monastir; ${ }^{3}$ Maxilla-Facial; ${ }^{4}$ Rheumatology, Taher Sfar Mahdia, mahdia, Tunisia

Background: Osteoporosis is defined as a skeletal disorder characterized by lowbone mass predisposing to an increased risk of fracture. At present, the evaluation of bone mineral density (BMD) is the main diagnostic tool in osteoporosis, and it is the most effective and accurate measurement for identifying and monitoring the disease. However, several studies have been conducted with the object of detecting whether these skeletal changes in the mandible are specific to the osteoporotic stage.

Objectives: The purpose of this study was to determine whether the madibular indices on panoramic radiographs are useful for identifying women with osteoporosis or osteopenia (low BMD).

Methods: Tunisian women aged from 30 to 60 years, who consulted the Rheumatology department of Fattouma Bourguiba university hospital in2017. The measure the BMD using the dual-energy X-ray absorptiometry (DEXA), were recruited to participate in this case-control study.Among the 60 women selected, 30 were diagnosed with osteoporosis or osteopenia (T-score $<-1$; cases) and 30 with normal T-score (T-score $>-1$; controls). The mandibular cortical index $(\mathrm{MCl})$, the mental index $(\mathrm{MI})$, the panoramic mandibular index (PMI) and the alveolar crest resorption degree (M/M ratio)were measured from digital panoramic radiographs in the right and left mandibles and the mean was calculated for each subject. The $\mathrm{MI}, \mathrm{PMI}$ and $\mathrm{M} / \mathrm{M}$ ratio values were evaluated using the $\mathrm{Z}$ test, and $\mathrm{MCl}$ values were analysed using the $\mathrm{Chi}^{2}$ test.

Results: The mean age of patients with osteoporosis/osteopenia was $58.61 \pm 8.11$ and $56.07 \pm 9.72$ in the control group. The mean bone miniral density (BMD)in vertebral site was $0.856 \pm 0.090 \mathrm{~g} / \mathrm{cm}^{2}$ and $1.216 \pm 0.185 \mathrm{~g} / \mathrm{cm}^{2}$ in control group.In femoral site, it was $0.877 \pm 0.221 \mathrm{~g} / \mathrm{cm}^{2}$ and $1.061 \pm 0.142 \mathrm{~g} / \mathrm{cm}^{2}$ respectively. The mean T-score in vertebral site was $-2.38 \pm 0.81 \mathrm{DS}$ in osteoporosis/ osteopenia group and $0.64 \pm 1.58 \mathrm{DS}$ in control group.In femoral site, it was $-1.57 \pm 0.97 \mathrm{DS}$ and $0.21 \pm 1.16$ respectively.

The analysis of the panoramic radiography showed that in the osteoporosis/osteopenia group: the mean value of $\mathrm{MI}$ was $3.56 \pm 0.89$, the mean PMI was $0.25 \pm 0.06$ and the mean M/Mratio was $0.71 \pm 0.14$; concerning the $\mathrm{MCl}: 46.7 \%$ were classified $\mathrm{C} 2$ stage and $53.3 \%$ were classified C3 stage.

In the control group, the mean $\mathrm{MI}$ was $4.42 \pm 0.98$, the mean $\mathrm{PMI}$ was $0.30 \pm 0.07$ and the mean $\mathrm{M} /$ Mratio was $0.77 \pm 0.11 ; 30.1 \%$ were classified $\mathrm{C} 1$ stage, $53.3 \%$ were classified C2 stage and only $16.6 \%$ were classified C1 stage.

This study showed that the $\mathrm{Ml}$ and the $\mathrm{PMI}$ were significantly smaller in the group with osteoporosis/osteopenia. However, the $\mathrm{M} / \mathrm{M}$ ratio was not significantly different.Therefore, the $\mathrm{MCl}$ was significantly more affected in the osteoporosis/osteopenia group.

Conclusions: In our study, we proved that the $\mathrm{MI}, \mathrm{PMI}$ and $\mathrm{MCl}$ values were affected in women with osteoporosis/osteopenia, compared with normal patients. Therefore, these indices could be used as an ancillary method in the diagnosis of osteoporosis in women.

\section{References:}

[1] C S Valerio et al, Dentomaxillofacial Radiology (2013) 42, 20120059.

[2] André Ferreira Leite, et al, Oral Surg Oral Med Oral Pathol Oral Radiol Endod 2011;112:648-656.

[3] Vlasiadis Z. Konstantinos, et al, Maturitas 58 (2007) 226-235.

Disclosure of Interest: None declared

DOI: 10.1136/annrheumdis-2017-eular.3558

\section{AB0849 ASSOCIATION BETWEEN PERIODONTITIS AND OSTEOPOROSIS}

M. Brahem ${ }^{1}$, M. Jguirim ${ }^{1}$, M. Khemis ${ }^{2}$, E. Chebil ${ }^{3}$, D. Chaabouni ${ }^{2}$, M. Younes $^{4}$, T. Ben Alaya ${ }^{2}$, M. Ben Khelifa ${ }^{2}$, I. Bejia ${ }^{1}$, M. Touzi ${ }^{1}$, S. Zrour ${ }^{1}$, N. Bergaoui ${ }^{1}$. ${ }^{1}$ Rheumatology; ${ }^{2}$ Stomatology; ${ }^{3}$ E.N.T. Head Neck Surgery, Fattouma Bourguiba Hospital Monastir, Monastir; ${ }^{4}$ Rheumatology, Taher Sfar Mahdia, mahdia, Tunisia

Background: Both periodontitis and osteoporosis have similar sign of bone resorption in nature and have multifactorial etiologic factors, although the mediator factors or mechanism may be different. How to prevent and treat these two bone-loss diseases is always an important issue in public health. However, the relationship of them is still uncertain.

Objectives: The aim of our study is to evaluate the relationship between periodentitis and osteoporosis.

Methods: Tunisian women aged from 30 to 60 years, who consulted the Rheumatology department of Fattouma Bourguiba university hospital between Febrary to December 2017. The measure the bone mineral density (BMD) using the dual-energy X-ray absorptiometry (DEXA), were recruited to participate in this case-control study. Among the 60 women selected, 30 were diagnosed with osteoporosis or osteopenia (T-score $<-1$; cases) and 30 with normal T-score (T-score $>-1$; controls). The oral examination was done by a dentist in the stomatology department in the same hospital.

Results: The mean age of patients with osteoporosis/osteopenia was $58.61 \pm 8.11$ 
[51-81 and 56.07 $\pm 9.72[40-76]$ in the control group.the mean bone miniral density (BMD)in vertebral site was $0.856 \pm 0.090 \mathrm{~g} / \mathrm{cm}^{2}$ and $1.216 \pm 0.185 \mathrm{~g} / \mathrm{cm}^{2}$ in control group. In femoral site, it was $0.877 \pm 0.221 \mathrm{~g} / \mathrm{cm}^{2}$ and $1.061 \pm 0.142 \mathrm{~g} / \mathrm{cm}^{2}$ respectively. The mean $\mathrm{T}$-score in vertebral site was $-2.387 \pm 0.814$ DS in osteoporosis/ osteopenia group and $0.643 \pm 1.587$ DS in control group. In femoral site, it was $-1.577 \pm 0.970 \mathrm{DS}$ and $0.213 \pm 1.162$ respectively. The oral examination showed an excessive tooth mobility in $60 \%$ and $36.7 \%$ of controls without a significant difference, a gingival recession in $50 \%$ and $30 \%$ of controls, the presence of periodontal pockets in $23.3 \%$ and $16.7 \%$ of controls without a significant difference, a plaque index $\geq 2$ in $53,3 \%$ of osteoporosis/osteopenia patients and $63,3 \%$ of controls and a non rectilinear trajectory of mouth opening in $13,3 \%$ and $3,3 \%$ of controls.

Conclusions: Our study showed that patients with osteoporosis or osteopenia have a poor oral hygiene, but without significant difference with control group.However, patients who were diagnosed as osteoporosis must pay more attention to their periodontal health. Good oral hygiene maintenance might be a crucial factor for preventing the deterioration of osteoporosis progressing.

\section{References:}

[1] Yi-Fang Huang et al, The Impact of Oral Hygiene Maintenance on the Association Between Periodontitis and Osteoporosis; Medicine; Volume 95, Number 6, February 2016

[2] Reddy MS. Osteoporosis and periodontitis: discussion, conclusions, and recommendations. Ann Periodontol. 2001;6:214-217.

Disclosure of Interest: None declared

DOI: 10.1136/annrheumdis-2017-eular.5184

\section{AB0850 HELICOBACTER PYLORI INFECTION AND OSTEOPOROSIS IN POST MONOPAUSAL WOMEN}

N. Abdolahi, M. Aghaei, M. Naghdi. Golestan Rheumatology Research Center, Golestan University of Medical Sciences, Gorgan, Iran, Islamic Republic Of

Background: Osteoprosis is a health problem that if is left untreated,can lead to serious health \& economical complications.It is also very common in postmenopausal women. Mineral deficiency, smoking, low BMI, some certain diseases and some medications can also cause osteoprosis. H.Pylori infection can also increase levels of inflammatory cytokines and bone turn over regulatory cytokines,as a result, it is likely that $\mathrm{H}$. pylori infected patients are at increased risk for osteoprosis.

Objectives: the present study was done to understanding the association between H.Pylori seropositivity and osteoprosis in postmenopausal women.

Methods: The study population consisted of 34 osteoporotic patients and 73 healthy controls. Serum levels of h.pylori antibody $(\mathrm{IgA}, \lg G)$ were measured by ELISA method.

Results: There was no difference in levels of $\lg A$ and $\lg G$ h.pylori antibody between patients and healthy controls

IgA seropositivity was $70.6 \%$ in osteoporotic women and $54.8 \%$ in healty women ( $p$ value: 0.1 ). Also $\mathrm{H}$. pylori IgG seropositivity was $82 \%$ in osteoporotic women and $75.3 \%$ in healthy women ( $\mathrm{p}$ value: 0.4 ). We didnot find any correlation between $\mathrm{H}$. pylori seropositivity and bone mineral density in post menopausal women.

Conclusions: In this study, we found that Helicobacter pylori infection does not increase the osteoporotic chance and is not a reliable risk factor for osteoporosis. greater sampele size, gastric biopsy to detect atrophic gastritis, bone turn over factors and Cag detection is recommended in order to achieve more accurate results

\section{References:}

[1] Shih-Chun Lin, Malcolm Koo, and Kun-Wei Tsai. Association between Helicobacter pylori Infection and Risk of Osteoporosis in Elderly Taiwanese Women with Upper Gastrointestinal Diseases: A Retrospective Patient Record Review, Gastroenterology Research and Practice, 2014:2014:814756.

[2] Kakehasi A.M, Mendes C.M.C, Coelho L.G.V, et al "The presence of Helicobacter pylori in postmenopausal women is not a factor to the decrease of bone mineral density," Arquivos de Gastroenterologia, vol. 44, no. 3, pp. 2007,266-270.

[3] Figura N, Gennari.L, Merlotti et al., "Prevalence of Helicobacter pylori infection in male patients with osteoporosis and controls," Digestive Diseases and Sciences, vol. 50, no. 5, pp. 2005, 847-852.

[4] Asaoka D, Nagahara A, Hojo M, Sasaki H, Shimada Y, Yoshizawa T, Osada T, Watanabe S. The Relationship between H. pylori Infection and Osteoporosis in Japan. Gastroenterol Res Pract. 2014;2014:340765.

Disclosure of Interest: None declared

DOI: 10.1136/annrheumdis-2017-eular.6445

\section{AB0851 ZOLEDRONATE AUDIT - ARE WE MEETING EUROPEAN GUIDELINES?}

N. Begum ${ }^{1}$, A. Khan ${ }^{1}$, A. Nandagudi ${ }^{2} .{ }^{1}$ Rheumatology/General Medicine; ${ }^{2}$ Rheumatology, Basildon \& Thurrock NHS Foundation Trust, Basildon, United Kingdom

Background: Zoledronate is recommended by European guidelines for the treatment of osteoporosis particularly where first-line oral drugs are ineffective or contraindicated. EULAR's quidelines are complimented by UK organisations including the National Institute of Health and Care Excellence (NICE), National Osteoporosis Guidelines Group (NOGG), British Society of Rheumatology (BSR) and the National Osteoporosis Society (NOS).

Objectives: The aim of our audit was to ascertain whether our use of zoledronate was compliant with current guidelines and review the real life experience.

Methods: We performed a retrospective audit of fifty patients who were commenced on zoledronate for the treatment of osteoporosis during 2012-2016 in our Trust. Data gathered included the reasons for commencement, whether patients had appropriate monitoring and the effect it had on DEXA and FRAX scores.

Results: The age ranged from 44-88 years; $67 \%$ were between $60-80$ years with $80 \%$ females. Vertebral fragility fractures were the most common type of fracture $(42 \%)$. Zoledronate was commenced primarily because of either intolerance or inefficacy to oral anti-osteoporotic treatment (Table 1). It was commenced as first line in $20 \%$ because of contraindications to oral drugs. Almost $70 \%$ of our patients received zoledronate for two or three years. There was an improvement by $43 \%$ and $38 \%$ in the DEXA t-score for the spine and hip respectively. Stable t-scores were recorded for the spine and hip in $49 \%$ and $54 \%$ respectively, whereas $8 \%$ deteriorated. Three patients sustained a fragility fracture and a further 11 experienced side effects (Table 2); five patients consequently stopped treatment. Only $3 \%$ had recorded FRAX scores pre- or post-zoledronate treatment. All of our patients had their calcium and renal function measured before each zoledronate infusion whilst over $80 \%$ had their vitamin D checked. All of our patients had dental checks prior to treatment. Following post-treatment DEXA scans $46 \%$ continued zoledronate and $16 \%$ were on a drug-free holiday. A third were switched to denosumab due to ineffectiveness, side effects or contraindications.

Table 1. Rationale for Zoledronate Commencement

\begin{tabular}{lcc}
\hline Rationale & Number & $\%$ \\
\hline Intolerance & 24 & $38 \%$ \\
GI Contraindication & 11 & $17 \%$ \\
Inefficacy & 24 & $38 \%$ \\
Other Contraindication & 4 & $7 \%$ \\
\hline
\end{tabular}

Table 2. Side Effects of Zoledronate

\begin{tabular}{lcc}
\hline Side Effect & Number & $\%$ \\
\hline Bony Pain & 1 & $9 \%$ \\
Flu-Like Symptoms & 5 & $46 \%$ \\
Aches & 1 & $9 \%$ \\
Acid-Reflux & 1 & $9 \%$ \\
Rash & 1 & $9 \%$ \\
Other & 2 & $18 \%$ \\
\hline
\end{tabular}

Conclusions: The majority of our patients had improvements or stability in bone mineral density T-scores with only $20 \%$ experiencing side effects. Our results show that the vast majority of our patients are treated with zoledronate in concordance with guidelines. Nonetheless we can make improvements in recording FRAX scores and monitoring vitamin $D$ levels. This has been highlighted to the multidisciplinary osteoporosis team and changes have been instigated. We plan to re-audit in due course.

References:

[1] Kanis JA, McCloskey EV, Johansson H, Cooper C, Rizzoli R, Reginster JY. European guidance for the diagnosis and management of osteoporosis in postmenopausal women J. ESCEO \& IOF. Osteoporos Int. 2013; 24: 23-57.

[2] Osteoporosis: Clinical guideline for prevention and treatment. NOGG. 2013.

Disclosure of Interest: None declared

DOI: 10.1136/annrheumdis-2017-eular.5015

\section{AB0852 INCREASED INFECTION RATE WITH CONCOMITANT RANK LIGAND INHIBITOR DENOSUMAB AND BIOLOGIC THERAPIES FOR RHEUMATIC DISEASES: REALITY OR ILLUSION? EXPERIENCE WITH 40 PATIENTS OVER 66 MONTHS AT THE UNIVERSITY OF SOUTHERN CALIFORNIA}

P.S. Chhibar, G. Ehresmann. Rheumatology, University of Southern California, Los Angeles, United States

Background: Patients with autoimmune diseases are at increased risk of early onset osteoporosis due to multiple reasons including prolonged exposure to corticosteroids and the disease process itself in RA patients. Same patients are more likely to be on TNF inhibitors or other biologics, which causes them to be at an increased risk of infections. Denosumab, an anti-RANK ligand inhibitor, itself a biologic, used to treat osteoporosis, is associated with increased infection risk as Receptor activator of nuclear factor kappa B ligand (RANKL) is also expressed on activated $T$ and $B$ lymphocytes (1). It is unknown if there is an added risk of infections when TNF inhibitors/biologic agents and denosumab are used concomitantly.

Objectives: To determine if denosumab and biologics are associated with increased infection risk.

Methods: Data was collected and analyzed on 40 patients in the rheumatology clinic who had been on denosumab and TNF inhibitor/ other biologic for 66 months at the Keck Medical Center of USC. 